\title{
A FAMILY OF LACUNARY RECURRENCES FOR FIBONACCI NUMBERS
}

\author{
CRISTINA BALLANTINE AND MIRCEA MERCA
}

Received 14 February, 2018

\begin{abstract}
We introduce an infinite family of lacunary recurrences for the Fibonacci numbers and give a combinatorial proof. The first entry in the family was proved by Lucas in 1876 .

2010 Mathematics Subject Classification: 11B39; 11B37

Keywords: Fibonacci numbers, recurrences
\end{abstract}

\section{INTRODUCTION}

A lacunary recurrence for a given sequence is a recurrence relation involving only terms of the sequence with indices in an arithmetic progression. We refer to the common difference in the indices in arithmetic progression as the gap of the lacunary recurrence. Lacunary recurrences allow for faster computation of sequence terms. The speed of the computation increases with the size of the gap. Lacunary recurrences have been studied for many types of sequences including Bernoulli numbers $[1,8,9,11]$, Euler numbers [8], $k$-Fibonacci numbers (which are Fibonacci polynomials at positive integer values) [4], Eisenstein series [10], Tribonacci numbers [6], more general sequences that include Bernoulli, Euler, Fibonacci and Genocchi numbers [5], and sequences satisfying an arbitrary linear recurrence [3, 12,13].

In this article we provide an infinite family of lacunary recurrences for Fibonacci numbers.

By definition, the first two Fibonacci numbers are 0 and 1 , and each subsequent number is the sum of the previous two. Thus, the sequence $\left\{F_{n}\right\}_{n \geqslant 0}$ of Fibonacci numbers is defined by the recurrence relation

$$
F_{n}=F_{n-1}+F_{n-2}
$$

with seed values $F_{0}=0$ and $F_{1}=1$. In this article, we set $F_{n}=0$ for $n<0$.

Lucas proved in 1876 that for every positive integer $n$ the Fibonacci numbers of even indices can be expressed in terms of the previous Fibonacci numbers of odd

This work was partially supported by a grant from the Simons Foundation (\#245997 to Cristina Ballantine). 
indices

$$
F_{2 n}=\sum_{k=1}^{n} F_{2 k-1}
$$

and vice versa

$$
F_{2 n+1}=1+\sum_{k=1}^{n} F_{2 k}
$$

After easy manipulations, one can rewrite the relations (1.1) and (1.2) as

$$
F_{n}=\frac{1+(-1)^{n}}{2}+F_{n-2}+\sum_{k=1}^{\left\lfloor\frac{n-1}{2}\right\rfloor} F_{n-2 k}
$$

This identity involves only indices in an arithmetic progression of length 2 and is thus a lacunary recurrence of gap 2 .

We generalize Lucas's identity (1.3) to lacunary recurrences with gap of any size for the Fibonacci numbers.

Theorem 1. Given a positive integer $N \geqslant 2$, we have

$$
F_{n}=F_{N} \cdot F_{N-1}^{\left\lfloor\frac{n-1}{N}\right\rfloor-1} \cdot F_{(n-1) \bmod N}+F_{N+1} \cdot F_{n-N}+F_{N}^{2} \cdot \sum_{k=2}^{\left\lfloor\frac{n-1}{N}\right\rfloor} F_{N-1}^{k-2} \cdot F_{n-k N},
$$

for all $n \geqslant N$.

As a consequence of this theorem, we have the following congruence identity.

Corollary 1. For a fixed integer $N \geqslant 2$ we have

$$
F_{n}-F_{N+1} \cdot F_{n-N} \equiv 0 \quad\left(\bmod F_{N}\right)
$$

for all $n \geqslant N$.

Using the relation [2, p. 4, Identity 3]

$$
F_{n}-F_{N+1} \cdot F_{n-N}=F_{N} \cdot F_{n-N-1},
$$

Theorem 1 can be written as follows.

Corollary 2. For $n \geqslant 0, N \geqslant 2$,

$$
F_{n+N}=F_{n \bmod N} \cdot F_{N-1}^{\lfloor n / N\rfloor+1}+F_{N} \cdot \sum_{k=0}^{\lfloor n / N\rfloor} F_{N-1}^{k} \cdot F_{n+1-k N} .
$$

If $N=2$, we recover Lucas's identities in the concise form (1.3).

If $N=3$, we recover the result of [4] (case $k=1$ of Corollary 5, part (2)):

$$
F_{3 n}=2 \sum_{k=1}^{n} F_{3 k-2}
$$




$$
\begin{aligned}
& F_{3 n+1}=1+2 \sum_{k=1}^{n} F_{3 k-1}, \\
& F_{3 n+2}=1+2 \sum_{k=1}^{n} F_{3 k} .
\end{aligned}
$$

For $N=4$ the statement of Theorem 1 becomes

$$
F_{n}=3 \cdot 2^{\left\lfloor\frac{n-1}{4}\right\rfloor-1} \cdot F_{(n-1) \bmod 4}+5 \cdot F_{n-4}+3^{2} \cdot \sum_{k=2}^{\left\lfloor\frac{n-1}{4}\right\rfloor} 2^{k-2} \cdot F_{n-4 k}
$$

and thus $F_{n}-5 F_{n-4} \equiv 0(\bmod 3)$.

\section{A COMBINATORIAL PROOF OF THEOREM 1}

In [7], Fibonacci numbers are interpreted combinatorially in terms of tilings of a $2 \times n$ grid with dominos. The author then provides combinatorial proofs for several identities, including Lucas's identities mentioned in the introduction. In particular, for $n \geqslant 1$, the $(n+1)$ st Fibonacci number, $F_{n+1}$, equals the number of distinct domino tilings of the $2 \times n$ grid. The empty $2 \times 0$ grid has, by convention, one tiling. For example, if $n=1$ there is only $F_{2}=1$ such tiling.

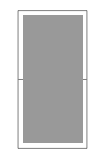

If $n=2$, there are $F_{3}=2$ tilings.

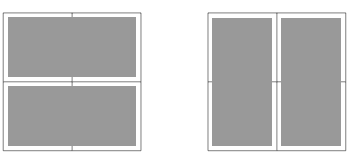

If $n=3$, there are $F_{4}=3$ tilings.
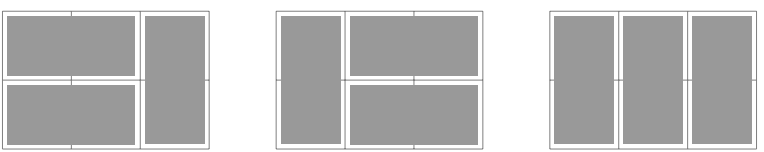

Obviously, the first row in a tiled $2 \times n$ grid completely determines the second row and the combinatorial interpretation given above is equivalent to the interpretation in [2] given in terms of tilings of the $1 \times n$ grid by (horizontal) dominos and squares. For our proof we will use the above interpretation in terms of domino tilings of the $2 \times n$ grid.

We denote by $a_{n}$ the number of distinct domino tilings of the $2 \times n$ grid. As mentioned above, we set $a_{0}=1$. Then $a_{n}=F_{n+1}$. 
Definition 1. The position of a domino in a tiling is defined as the column number $i$ of its most left box.

For example, the position of the shaded horizontal domino in the figure below is 4 while the position of the vertical domino is 2 .

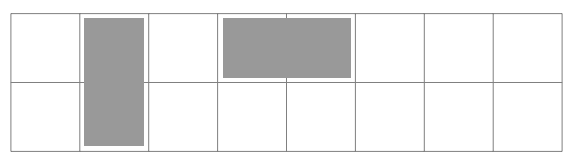

We will never have to refer to the position of horizontal dominos in the second row as they are determined by the horizontal dominos in the first row.

To prove Theorem 1, we count the domino tilings of the $2 \times n$ grid according to whether there is a horizontal domino with position $N$ or not.

The number of tilings of the $2 \times n$ grid with no horizontal domino with position $N$ equals $a_{N} \cdot a_{n-N}$ since in this case the left $2 \times N$ grid can be tiled independently of the right $2 \times(n-N)$ grid.

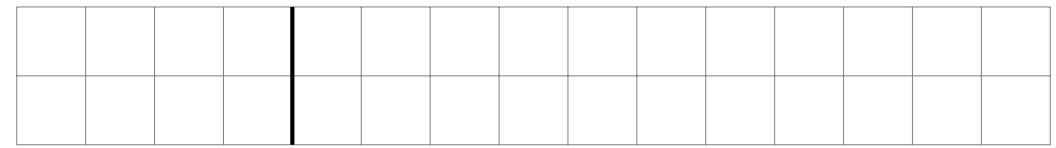

If there is a horizontal domino with position $N$, by the same argument, there are $a_{N-1} \cdot a_{n-N-1}$ such tilings.

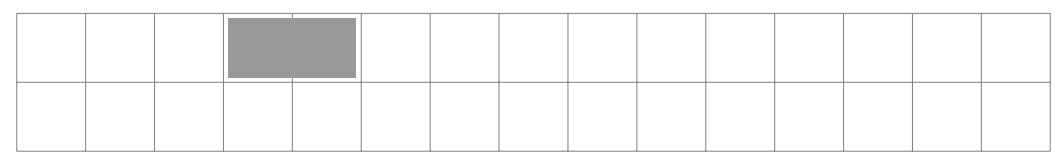

Thus,

$$
a_{n}=a_{N} \cdot a_{n-N}+a_{N-1} \cdot a_{n-N-1} .
$$

Next, we count the tilings of the $2 \times(n-N-1)$ grid in a similar manner according to whether there is a horizontal domino with position $N-1$ or not. We have

$$
a_{n-N-1}=a_{N-1} \cdot a_{n-2 N}+a_{N-2} \cdot a_{n-2 N-1}
$$

and thus

$$
a_{n}=a_{N} a_{n-N}+a_{N-1}\left(a_{N-1} a_{n-2 N}+a_{N-2} a_{n-2 N-1}\right) .
$$

Repeating the procedure, and using the fact that

$$
a_{n-k N-1}=a_{N-1} a_{n-(k+1) N}+a_{N-2} a_{n-(k+1) N-1},
$$

for all $1 \leqslant k \leqslant\left\lfloor\frac{n}{N}\right\rfloor-1$, we have

$$
\begin{aligned}
a_{n}= & a_{N} a_{n-N}+a_{N-1}\left(a_{N-1} a_{n-2 N}+a_{N-2}\left(a_{N-1} a_{n-3 N}+a_{N-2}\left(a_{N-1} a_{n-4 N}\right.\right.\right. \\
& \left.\left.\left.+\cdots+a_{N-2}\left(a_{N-1} a_{n-N\left\lfloor\frac{n}{N}\right\rfloor}+a_{N-2} a_{n-1-N\left\lfloor\frac{n}{N}\right\rfloor}\right) \cdots\right)\right)\right)
\end{aligned}
$$




$$
=a_{N} a_{n-N}+\sum_{k=2}^{\left\lfloor\frac{n}{N}\right\rfloor} a_{N-1}^{2} a_{N-2}^{k-2} a_{n-k N}+a_{N-1} a_{N-2}^{\left\lfloor\frac{n}{N}\right\rfloor-1} a_{n-1-N\left\lfloor\frac{n}{N}\right\rfloor} .
$$

\section{CONCLUding REMARKS}

An infinite family of lacunary recurrence formulas for Fibonacci numbers has been introduced in this paper using a combinatorial approach. Some specializations of this result are also presented. The first entry in this family is well known as Lucas's identity (1.3). The second entry is also known [4].

We conclude with an open problem. The Lucas numbers are defined by $L_{0}=2$, $L_{1}=1$ and for $n>1$,

$$
L_{n}=L_{n-1}+L_{n-2} \text {. }
$$

These numbers are related to the Fibonacci numbers by the identity

$$
L_{n}=F_{n-1}+F_{n+1} \text {. }
$$

It is natural to ask for a general lacunary recurrence for Lucas numbers in the spirit of Theorem 1.

\section{REFERENCES}

[1] T. Agoh and K. Dilcher, "Convolution identities and lacunary recurrences for Bernoulli numbers," J. Number Theory, vol. 124, no. 1, pp. 105-122, 2007, doi: 10.1016/j.jnt.2006.08.009.

[2] A. T. Benjamin and J. J. Quinn, Proofs that really count. The art of combinatorial proof The Dolciani Mathematical Expositions, 27. Washington, DC: Mathematical Association of America, 2003.

[3] D. Birmajer, J. B. Gil, and M. Weiner, "Linear recurrence sequences with indices in arithmetic progression and their sums," Integers, vol. 16, pp. Paper No. A71, 13 pp., 2016.

[4] S. Falcon and A. Plaza, "On $k$-Fibonacci numbers of arithmetic indexes," Appl. Math. Comput., vol. 208, no. 1, pp. 180-185, 2009, doi: 10.1016/j.amc.2008.11.031.

[5] F. T. Howard, A general lacunary recurrence formula Applications of Fibonacci numbers, 9. Dordrecht: Kluwer Acad. Publ., 2004.

[6] N. Irmak and M. Alp, "Tribonacci numbers with indices in arithmetic progression and their sums," Miskolc Math. Notes, vol. 14, no. 1, pp. 125-133, 2013, doi: 10.18514/MMN.2013.523.

[7] M. Krzywkowski, "New Proofs of Some Fibonacci Identities," International Mathematical Forum, vol. 5, no. 18, pp. 869-874, 2010.

[8] D. H. Lehmer, "Lacunary recurrence formulas for the numbers of Bernoulli and Euler," Ann. of Math. (2), vol. 36, no. 23, pp. 637-649, 1935, doi: 10.2307/1968647.

[9] M. Merca, "On lacunary recurrences with gaps of length four and eight for the Bernoulli numbers," Bull. Korean Math. Soc., vol. 56, no. 2, pp. 491-499, 2019, doi: 10.4134/BKMS.b180347.

[10] M. H. Mertens and L. Rolen, "Lacunary recurrences for Eisenstein series," Res. Number Theory, vol. 1, no. Art. 9, p. 5 pp., 2015, doi: 10.1007/s40993-015-0010-x.

[11] S. Ramanujan, "Some properties of Bernoulli's numbers," J. Indian Math. Soc., vol. 3, pp. 219234, 1911.

[12] A. G. Shannon, "Some lacunary recurrence relations," Fibonacci Quart., vol. 18, no. 1, pp. 73-79, 1980.

[13] P. T. Young, “On lacunary recurrences,” Fibonacci Quart., vol. 41, no. 1, pp. 41-47, 2003. 
Authors' addresses

\section{Cristina Ballantine}

College of The Holy Cross, Department of Mathematics and Computer Science, Worcester, MA 01610, USA

E-mail address: cballant@holycross.edu

\section{Mircea Merca}

Academy of Romanian Scientists, Ilfov 3, Sector 5, Bucharest, Romania

E-mail address: mircea.mercalprofinfo.edu.ro 